\title{
Entwicklungen im Court Management in vergleichender Sicht
}

Rosemarie Barberet und Ramón y Cajal

\section{Unabhängigkeit der Justiz und Court Management}

is vor etwa zwanzig Jahren wurde das Wort "Management « an Gerichten nicht oft in den Mund genommen. »Management « erschien eher als ein Begriff, der zum Bereich der Exekutive und nicht zum Bereich der Judikative gehörte. Gerichte sahen sich als neutrale Entscheidungsträger und nicht als Manager von Fällen. Die Vorstellung, Fälle zu managen, erschien weit entfernt vom Verfolgen vorgeschriebener rechtlicher Verfahrensschritte, ja fast im Gegensatz dazu. Überdies sind Richterinnen und Richter ${ }^{1}$ nie zu Managern ausgebildet worden. Als professionelle Rechtsanwender sehen sich die meisten über den »banalen « Dingen des Managements stehend. Unter jenen jedoch, die sich mit der Modernisierung der Gerichtsbarkeit beschäftigen, herrscht die verbreitete Ansicht, dass Court Management den Schlüssel zur Unabhängigkeit der Justiz darstellt. Wenn es den Gerichten nicht gelinge, ihre internen Abläufe gut zu managen und das in transparenter Art und Weise, würden sie anfällig werden für die Einflussnahme durch die anderen Gewalten.

Dieser Aufsatz will eine Übersicht über Strömungen im Court Management geben. Er ist kein erschöpfender Literaturüberblick, sondern versucht - insbesondere mit Seitenblick auf Forschung und politische Akteure im deutschsprachigen Raum - eine Vorstellung von innovativen Entwicklungen und Ergebnissen in verschiedenen Regionen der Welt zu vermitteln, die Beachtung und Nachahmung verdienen könnten.

\section{Court Management im Kontext allgemei- ner Trends in der Gerichtsverwaltung}

Die meisten Beobachter stimmen darin überein, dass Gerichtsorganisation eine sperrige Angelegenheit ist. Viele Gerichte sind »Lehen« (Derr, 2001: 224) einzelner Richter, die von Dienstleistern umgeben sind, die sie nicht mit anderen Richtern gemeinsam in Anspruch nehmen. Es gibt keine klare Hierarchie außer derjenigen, die der Instanzenzug vorgibt. Es gibt kaum Management oder auch nur Koordination von Leistungen, keine Festlegung von Zielen und kein Leitbild der Organisation. Gerichtsakteure gehen davon aus, dass es materielle und verfahrensrechtliche Bestimmungen, ebenso wie langjährig gepflegte Traditionen der Rechtskultur sind, die die Gerichte in Gang halten. Und obwohl die meisten Richter Rechtsfindung nicht als endlosen Prozess auffassen, halten sie ihre Entscheidungen für Kunstwerke, deren Herstellung $\mathrm{zu}$ beschleunigen der Forderung nach schlampiger juristischer Arbeit gleichkäme.

Moderne Techniken des Court Management wären nicht wirklich möglich, hätten sich nicht strukturelle Veränderungen in der Gerichtsverwaltung ebenso wie eine neue Ideologie der Justizleistung entwickelt. Einstellungen und die Bereitschaft, Innovationen zu akzeptieren, ändern sich oft erst nach oder im Gleichschritt mit gesetzlichen und strukturelle Änderungen. Manchmal werden Einstellungsänderungen durch außenstehende Einrichtungen vermittelt - durch Geldgeber wie etwa die Weltbank, die Inter-Amerikanische Entwicklungsbank, US AID, das UK Department for International Development oder die Generaldirektion für Entwicklung der Europäischen Kommission. Diese Organisationen vertreten die Ansicht, dass Rechtsstaatlichkeit, mit gut geführten Gerichten als sichtbarem Teil davon, der Schlüssel zu sozio-ökonomischer Entwicklung ist und dass Gerichte effizient, effektiv, und transparent in ihren Tätigkeiten sein müssen. Ihr besonderes Augenmerk gilt oft den Bereichen des Zivilrechts und des Verwaltungsrechts. Manchmal befassen sie sich aber auch mit den Tätigkeiten von Strafgerichtshöfen oder dem gesamten Gerichtssystem eines Landes. In anderen Fällen sind es leitende Persönlichkeiten nationaler Gerichte oder Akteure der Politik, die sich diesen Konzepten verschreiben. In Großbritannien hat gewiss der »Managerialismus « als Regierungsideologie der Konservativen und später New Labours, dazu beigetragen, die Gerichtshöfe zu dem zu machen, was sie heute sind. Politische Ideologien, die die Justiz eher als eine Dienstleistung ansehen denn als eine eigene Macht oder eine privilegierte Institution, erleichtern wahrscheinlich die Entwicklung der Gerichte zu einer gut gemanagten Organisation.
Auch gute Forschung kann Einstellungsänderungen bewirken. Ostrom und Hanson (1999) stellen in ihrer wichtigen US-amerikanischen Studie Efficiency, Timeliness and Quality: A New Perspective from Nine State Criminal Trial Courts den gerichtlichen »common sense « in Frage, dass die Beschleunigung der Entscheidungsfindung eine verminderte Qualität der richterlichen Entscheidungen mit sich bringt. Die Autoren zeigen, dass das Grundprinzip der Proportionalität (dass nämlich schwerwiegendere und kompliziertere Fälle mehr Zeit eines Gerichts in Anspruch nehmen als andere) auch vor den schnelleren Gerichten der Stichprobe galt. Geschwindigkeit ging nicht mit einem Abschied von der Aufmerksamkeit für Details einher. Es wurde kein »Justizfließband « konstatiert und Staatsanwälte und Verteidiger sahen einander nach wie vor als gegnerische Parteien an. Was die schnelleren Gerichtshöfe sehr wohl von den anderen unterschied, war eine effizientere Arbeitseinstellung von Strafverfolgern wie Anwälten. Während die Ressourcenknappheit überall vergleichbar war, empfanden die Rechtsanwender an den schnelleren Gerichtshöfen einander als gut vorbereitet, erfahren und qualifiziert (Ostrom and Hanson, 1999: 108-109).

\section{Management durch Zielvorgaben und die Entwicklung von Indikatoren der Gerichts- leistung}

Wenn die Justiz als eine öffentliche Dienstleistung aufgefasst wird, werden die Gerichte auch als Institutionen angesehen, die - wie andere auch - von Steuerzahlern bezahlt werden und als solche mit ihrem guten Service eine Gegenleistung für erhaltenes Geld liefern müssen. Im Rahmen einer Auffassung der Gerichte als Dienstleister bilden sich zwei zentrale Managementstrategien heraus: Management durch Zielvorgaben und die Entwicklung von Indikatoren der Gerichtsleistung.

Management durch Zielvorgaben erlaubt es den Gerichten, ein Leitbild oder Hauptziel festzulegen, kurzfristige und langfristige Ziele zu formulieren und Messinstrumente für die Gerichts- 
leistung $\mathrm{zu}$ entwickeln und anzuwenden, um Fortschritte festzustellen. Ein gutes Beispiel für einen derartigen Prozess stellt das Court Service of England and Wales dar ${ }^{2}$. Das Court Service definiert seine »Vision « folgendermaßen: [Das Court Service] "will Justizdienste von Weltklasse zur Verfügung stellen, ein Ort sein, an dem zu arbeiten die Belegschaft stolz ist, von [seinen] Kunden geschätzt werden, das Vertrauen der Allgemeinheit genießen und preiswert sein« (2002-2003 Court Service Annual Report). Die Zielsetzungen für das Jahr 2002-2003 für die Strafjustiz waren, »ein faires, rasches und effektives System der Strafjustiz zu sichern

(1) durch partnerschaftliche Zusammenarbeit mit anderen Behörden,

(2) indem durch die Anwendung von Fallmanagement die Effizienz verbessert wird und rechtzeitig Ergebnisse erreicht werden,

(3) indem den Bedürfnissen von Opfern, Zeugen, und Geschworenen in einer zunehmend heterogenen Gesellschaft entsprochen wird und dabei zugleich die Rechte der Angeklagten gewahrt werden und

(4) durch die Modernisierung von Gerichtsverfahren.

Das Court Service hat in Zusammenhang mit diesen Zielsetzungen eine Anzahl von entscheidenden Leistungsindikatoren festgelegt: Der Prozentsatz der Beschuldigten/in Berufung Gehenden, deren Fälle innerhalb der geplanten Zeit verhandelt werden, die Verbesserung des Court Service nach dem Maßstab der European Foundation for Quality Management und die prozentuelle Verbesserung im Grad der Zufriedenheit der Gerichtsbenutzer. Für jeden der Leistungsindikatoren wird jährlich ein Ziel festgelegt. Der Jahresbericht des Court Service informiert darüber, ob das jeweilige Ziel erreicht wurde. Ein Unternehmensplan für das folgende Jahr wird aufgestellt und so weiter.

In föderalen Systemen, wie etwa den Vereinigten Staaten, ist es oft trotz bestehender Gemeinsamkeiten zwischen den Gerichten politisch nicht machbar, ein Leitbild für alle Gerichte aufzustellen. Dennoch ist es möglich, gewisse Zielsetzungen und Standards festzulegen, die durch eine Reihe von Indikatoren dargestellt werden können. Die United States Trial Court Performance Standards, die nach zehnjähriger Arbeit im Jahr 1997 fertig gestellt wurden, nennen fünf Elemente einer guten Gerichtsleistung:

(1) Zugang zum Recht

(2) expeditive, zeitgerechte Verfahrensdurchführung

(3) Gleichheit, Fairness und Integrität

(4) Unabhängigkeit und Rechenschaftspflichtigkeit

(5) Vertrauen der Allgemeinheit.

Insgesamt 22 Standards decken diese fünf Bereiche ab. Für jeden gibt es eine detaillierte Beschreibung, wie er zu messen ist. Dazu gehört die
Nutzung von Gerichtsstatistiken wie anderer Arten der Leistungsmessung, etwa die Beobachtung von Gerichtsverfahren und Umfragen unter Gerichtsbenutzern (Keilitz, 2000). Die Gerichtsleistungsstandards sind nicht verpflichtend. Es steht den Gerichten der Vereinigten Staaten frei, das System zu übernehmen.

Auch in nicht-englischsprachigen Ländern, deren Rechtskultur sehr verschieden von der angelsächsischen ist, sind Leistungsmessungsstandards aufgekommen. Es wäre ein Fehler anzunehmen, dass dafür eine angelsächsische Mentalität notwendig ist. Santos Pastor, dessen Arbeit auch in Spanien, Lateinamerika und der Karibik ihre Bedeutung hat, weist darauf hin, dass es - wiewohl bis dato kein »universelles « System von Standards existiert - einige gemeinsame Grundprinzipien zu geben scheint. Er identifiziert eine Reihe von Standards (»zwölf Gebote«), an denen Justizpolitik und Justizsystem evaluiert werden können: Unabhängigkeit, Unparteilichkeit, Kompetenz, Zugänglichkeit, Billigkeit (equity), Effizienz, Effektivität, Vermeiden/Reduktion von Verzögerungen, Transparenz, Verantwortlichkeit, Vorhersagbarkeit, und Qualität. (2003b: 28). Berechenbarkeit und Qualität sind zwei von Pastors »Geboten«, die sich nicht oft in anderen Leistungsmanagementsystemen finden. Sie spiegeln Pastors Vertrautheit mit internationaler Gerichtsreform sowie mit den Problemen von Gerichten in Entwicklungs- und Schwellenländern wieder. Vorhersagbarkeit Rechtssicherheit und Konsistenz - ist die wichtigste Zielsetzung in Hinblick auf die Erreichung von sozialer Stabilität und nachhaltiger Entwicklung. Die juristische Qualität von Entscheidungen wiederum wird bei der Leistungsmessung oft vernachlässigt. Die meisten Systeme von Leistungsindikatoren messen lediglich die Qualität der Dienstleistung. Wenn aber alle Aspekte von Gerichtssystemen evaluiert werden sollen und nicht nur diejenigen, für die das am einfachsten zu bewerkstelligen ist, muss diese Herausforderung bewältigt werden. Rechtsentscheide müssen von hoher Qualität sein - konsistent, gut argumentiert und gut begründet -, formen sie doch das Rückgrat eines effektiven Justizsystems.

\section{Techniken des Court Management}

Techniken des Court Management stehen in logischem Zusammenhang mit den verschiedenen organisatorischen Problemen, an denen Gerichtssysteme leiden. Bisweilen helfen Gerichtszusammenlegungen oder ehrgeizige Anstrengungen zu einer nationalen Gerichtsreform, grundlegende organisatorische Probleme zu lösen. Oft aber bestehen Probleme fort. Beispielsweise nennt die Kommission der Juristen der Andenregion (Comisión Andina de Juristas) folgende Defizite, die den Gerichten in der Region gemeinsam sind: Instabilität, Verzögerungen, mangelnde Koordination mit anderen staatlichen Einrichtungen, Mangel an Professionalität, an Follow-up-Proze- duren, Widerstand gegen Veränderungen, komplizierte, rigide und ritualisierte Verfahren, eine schwache institutionelle Basis, mangelnder $\mathrm{Zu}-$ gang zur Judikatur, Mangel an Unabhängigkeit, an Planung und Evaluation, an alternativen Streitbeilegungsmethoden, an Kontrolle über die richterliche Autorität, Korruption und unzureichende Leistungen von Gerichtsbediensteten (Comisión Andina de Juristas, 2002).

\section{Wie kann Court Management zur Lösung dieser Probleme beitragen?}

Court Management legt besonderen Wert auf Effizienz. Dazu werden allgemein übliche Management Techniken und aus dem Gebiet der Rechtsökonomie entlehnte Konzepte eingesetzt (Pastor, 2003a). Dazu gehören die Implementierung von Fallmanagement, die Konzentration auf Gerichtshilfsdienste, die Spezialisierung von Gerichtsaufgaben, Managementinformationssysteme und automationsunterstütztes Leistungsmanagement, Unterstützung für Selbsthilfemaßnahmen und Zugang zum Recht, die Einrichtung von Belohnungssystemen (incentives) für Gerichtsakteure, insbesondere Richter, und die Schaffung des "Gerichtsmanagers $\ll^{3}$. Es ist wichtig, dabei nicht $\mathrm{zu}$ vergessen, dass die Umstrukturierung von Gerichten weniger mit Personalaufstockungen und der Anschaffung neuer Computer zu tun hat als mit der Konzentration auf Zielvorgaben und der Rationalisierung von Aufgaben. In der Tat ist es oft die rigide Rollendefinition der Gerichtsbediensteten, die in manchen Ländern einer Managementreform im Weg steht. Bendala (2003) erinnert daran, dass es zuerst gilt, darüber nachzudenken, welche Arten von Produkten oder Dienstleistungen von den Gerichten gewünscht werden, ehe darangegangen wird, die Arbeitsweise der Gerichte umzustrukturieren.

\section{Fallmanagement}

Der Fallmanagementansatz ist möglicherweise das übergreifende Element des Court Management, das die Verwendung anderer Techniken und Methoden strukturiert. Der Grundgedanke von Fallmanagement ist der, dass die Gerichte allzu lange Fälle als passive Empfänger entgegengenommen und bearbeitet haben. Sie haben damit externen Akteuren, also Streitparteien mit ihren Anwälten, die Möglichkeit gegeben, Fälle zu verschleppen oder die Zeit des Gerichts ineffizient zu nutzen. Theoretisch erlauben Maßnahmen des Fallmanagements den Gerichtsakteuren, die Kontrolle über Fälle zu verbessern, indem sie mehr Herrschaft über den Verfahrensablauf gewinnen. Das mag einigermaßen undemokratisch oder zumindest "non-adversariell « klingen, es kann aber sowohl im Rahmen von Systemen mit einem Rechtsgegnerschafts- wie einem Anklageprinzip geschehen. Der Ansatz hat eine Ähnlichkeit mit neue Polizeistrategien, mit »problemfokussierter 
Polizeiarbeit«, die der Polizei mehr Kontrolle über ihre Arbeit bringt, weil nicht mehr so sehr das Sperrfeuer von Dienstleistungsanforderungen von Seiten der Allgemeinheit bestimmt, wie sie Verbrechen bekämpft.

Der Fallmanagementansatz beinhaltet Techniken, die es erlauben, Fälle effizienter zu behandeln. Beispielsweise werden Fälle, sobald sie aufgenommen werden, nach ihrer Komplexität analysiert und dann auf eine Bearbeitungsschiene gestellt. Differenziertes Court Management bedeutet, dass gleichartige Fälle von einem spezialisierten Gericht oder einem spezialisierten Richter behandelt werden und dass den komplexeren Fällen mehr Ressourcen und mehr Zeit gewidmet wird, sodass sie nicht zu einer Verzögerung der anderen in der Warteschlange führen. (Eine gute Evaluation von differenziertem Fallmanagement findet sich bei Taxman und Ellis 1999).

Automatisierte Informationssysteme bedeuten, dass Technologie eingesetzt werden kann, um Fälle besser zu verwalten, Fehler zu reduzieren und Arbeitskraft einzusparen. Wenn die Automatisierung erfolgreich ist, kann ein System von Leistungsindikatoren eingebaut werden und Feedback in Echtzeit liefern. Ein guter Fallmanagementansatz beruht auf Expertise im Gerichtswesen ebenso wie auf der Fähigkeit anderer Rechtsanwender und der Allgemeinheit, die Dienste von Gerichten vernünftig in Anspruch zu nehmen. Beispielsweise können Anwälte um eine Einschätzung der Zeit, die sie für eine Anhörung benötigen werden, gebeten werden und diese Schätzung kann für die Planung des Prozesskalenders des Gerichts herangezogen werden. Die Allgemeinheit kann ermutigt werden, ihre eigenen Fälle mit Hilfe automatisierter Systeme zu verfolgen, oder sich mit dem Gerichtssystem besser vertraut $\mathrm{zu}$ machen. $\mathrm{Zu}$ gleich muss es für die Gerichtsakteure Leistungsanreize geben. Spanische Richter erhalten derzeit leistungsorientierte Bezahlung auf der Grundlage der Fälle, die sie behandeln, nach Komplexität gewichtet. Es gibt keinen Grund, warum nicht auch andere Akteure bei Gericht ähnliche Anreize bekommen sollten. In England werden zum Beispiel Gerichtsbedienstete, die Telefonanrufe entgegennehmen, auf ihre Genauigkeit, die gebotene Hilfestellung und daraufhin beobachtet, welche Höflichkeit sie an den Tag legen.

Der Fallmanagementansatz hat auch seine Kritiker, aber ein Großteil der Kritik hat mit Implementierungsproblemen $\mathrm{zu}$ tun - insbesondere mit mangelnder Abstimmung zwischen einem Managementinformationssystem, der Rolle des Richters und den rechtlichen Vorschriften, einem Mangel an Schulung (siehe Plotnikoff und Woofson, 2002), oder einem Mangel an Verständnis für die Notwendigkeit einer bestimmten Dokumentation seitens des Gerichtspersonals, folglich einem Mangel an Motivation, die für ein gutes Funktionieren des Fallmanagementsystems notwendigen Daten einzugeben.
Der Gerichtsmanager wird in einem System wie dem hier beschriebenen unverzichtbar. Gerichtsbedienstete haben zwar eine derartige Rolle bis zu einem gewissen Grad inne, sie sind aber nicht in Management ausgebildet und sehen die Rolle des Managers selten als Mittelpunkt ihrer Tätigkeit. Nichtsdestoweniger variieren auch die Verantwortlichkeiten von Gerichtsmanagern beträchtlich. Das veranlasste die National Association for Court Management in den Vereinigten Staaten, Kompetenzanforderungen für Gerichtsmanager auf folgenden Gebieten zu definieren: Zweck und Verantwortlichkeiten von Gerichten, Management des »Umlaufs « an Fällen (case flow management), Führungsqualitäten, Entwicklung von Visionen und strategische Planung, Kommunikation zwischen Gericht und örtlicher Gesellschaft, Ressourcen, Budget und Finanzen, Personalwesen, Bildung, Aus-/Weiterbildung und Informationstechnologiemanagement. Die Vielfalt von Aufgaben, die Gerichtsmanagern übertragen werden, und die Professionalisierung spiegelt die zentrale Rolle dieser Figur für das Court Management wieder. Wiederum argumentieren viele, dass gerade zuzulassen, dass eine Figur mit solchen exekutivischen Anstrichen in die Gerichtsbarkeit eindringt, eine wichtige Garantie für die Unabhängigkeit der Judikative von den anderen Gewalten darstellt.

\section{Court Management an Strafgerichten und die Theorie offener Systeme: Reduktion von Rückfallsraten als grundlegende Technik des Court Management}

Die vielleicht jüngste Innovation an den Gerichtshöfen sind Programme, die auf die Reduktion von »Rückfällen « abzielen und dabei den Gerichten und Richtern eine Schlüsselrolle zuweisen. Einige Autoren betrachten diese Entwicklung als natürliche Folge von Fallmanagementsystemen (Taxman und Ellis, 1999), während andere sie als völlig neu und unabhängig von der Bewegung für ein gutes Gerichtsmanagement ansehen. Wenn man Gerichte als offene Systeme ansieht, die ihre Umgebung beeinflussen aber auch von ihr beeinflusst werden, dann stellt die Einschränkung der von außen kommenden Nachfrage nach Justizleistungen jedenfalls ein wichtiges Managementinstrument dar. In der Tat hat die Literatur zum Court Management eine Tendenz, in erster Linie die klassischen Formen von Maßnahmen zur Verringerung der Nachfrage nach Justizdiensten in Betracht $\mathrm{zu}$ ziehen: Alternative Streitschlichtung, Absprachen über das Strafmaß im Gegenzug zu einem Schuldbekenntnis des Angeklagten (plea bargaining), Privatisierung von Rechtsdiensten, Rechtsberatungszentren und Sammelklagen (Pastor, 1993).

Ich möchte argumentieren, dass die Verringerung von Rückfällen, d.h., sicherzustellen, dass bereits vom Gericht bearbeitete Fälle nicht noch einmal in das Gerichtssystem kommen, auch eine nützliche Technik des Court Management ist, in dem ineffizienter Input in das Gerichtssystem reduziert wird. Im folgenden werden rezente Innovationen im Bereich der Reduktion der Rückfallshäufigkeit diskutiert, bei denen Gerichte Protagonisten des Prozesses sind.

\section{Einbindung von Gerichten in Präventions- programme}

Herkömmliches kriminologisches Denken sieht Gerichte entweder als Instrumente der Abschreckung, bestenfalls als schöpferische Gestalter von Urteilen. Die Macht, Urteile zu sprechen, ist das Mittel, das Gerichten zur Verfügung steht, um einen Einfluss auf Kriminalität zu nehmen, indem sie die »Botschaft « an Straftäter schicken, ihr Verhalten sei nicht akzeptabel, zu bestrafen oder in anderer Weise zu behandeln. Darüberhinaus sind Richter üblicherweise nicht in hohem Maß in Präventionsprogramme in der Gesellschaft involviert. Sie halten auch nicht gewohnheitsmäßig Nachschau, wie weit von ihnen gefällte Urteile bei den Straftätern eine Änderung erzielen. Die Rolle der Richter in der Verbrechenseindämmung ist eine formeller Macht, hat aber relativ enge Grenzen. In Anbetracht der Kürze der Interaktion und der normalerweise bestehenden sozialen Distanz zwischen Richtern und Tätern erscheint es gewagt zu behaupten, dass die Interaktion zwischen Angeklagtem und Richter eine mit starker Wirkung sei.

\section{Verfahrensgerechtigkeit}

Dennoch stellen neue rechtspsychologische Perspektiven der "Verfahrensgerechtigkeit « (Tyler, 2003) und der »therapeutischen Justiz« (Winick und Wexler, 2003) diese überkommene Sichtweise von Gericht und Richtern in Frage. Nach diesen beiden Ansätzen kann, was bei Gericht passiert (und nicht nur das Resultat eines Gerichtsverfahrens) und zu Angeklagten und Opfern gesagt wird, eine beträchtliche Auswirkung darauf haben, wie der Täter eine Urteilsentscheidung aufnimmt und wie der Verarbeitungsprozess beim Opfer verläuft. Insbesondere argumentiert Tyler, dass es die empfundene Fairness des Verfahrens ist, die die Betroffenen $\mathrm{zu}$ entsprechenden Verhaltensmodifikationen motiviert. Gerichte können eine nachhaltige Befolgung von Gesetzen erreichen, indem sie sicher stellen, dass die Allgemeinheit ihre Arbeit als faire Ausübung von Autorität wahrnimmt.

Es liegt in der Macht von Gerichten, Entscheidungen mit Auswirkungen auf das Leben von Menschen zu treffen. Es ist aber wünschenswert, dass Menschen den rechtlichen Richtlinien auch dann folgen, wenn kein Richter anwesend ist und niemand Verhalten kontrolliert. Gerichte haben sich daher um die Kooperation der Menschen zu bemühen, die vor Gericht erscheinen. Beispielsweise kann ein Richter einem Ladendieb in drohender Weise mitteilen, er möge ein bestimmtes 
Geschäft nie wieder betreten. Aller Wahrscheinlichkeit nach werden seine Worte aber keinen anhaltenden Effekt haben, wenn nicht auch andere Elemente mit ins Spiel gebracht werden. Tyler argumentiert dass die Gerechtigkeit des Verfahrens (procedural justice) von größerer Bedeutung als die Gerechtigkeit der Entscheidung (distributive justice) für die Beachtung der Entscheidung durch das Publikum ist. Die Befunde von Tyler empfehlen Gerichten, weniger Augenmerk auf das »objektive " Ergebnis der Entscheidung als vielmehr auf das "subjektive« Maß der Verfahrensfairness für diejenigen zu legen, die von Gerichtsentscheidungen betroffen sind. Das liegt auch daran, dass es für Gerichte nahezu unmöglich ist, Entscheidungen zu treffen, die von jeder Partei als positiv empfunden werden. »Win-Win« Situationen bei Gericht sind nicht häufig. Nichtsdestotrotz streben Gerichte theoretisch nach Akzeptanz all ihrer Entscheidungen, ob sie den Betroffenen gefallen oder nicht.

Was macht die Verfahrensgerechtigkeit einer Entscheidung aus? Tyler nennt Faktoren wie empfundene Objektivität der Entscheidungsträger, respektvoller und ihre Rechte anerkennender Umgang mit Beteiligten, Verständlichkeit des Entscheidungsprozesses und Beteiligungsmöglichkeiten am Zustandekommen der Entscheidung. Die Implikationen für Court Management sind klar und haben viel damit zu tun, eine gute Kommunikation zwischen den Gerichtsakteuren und dem Publikum zu unterstützen.

\section{Therapeutische Justiz?}

Winick und Wexler (2003) gehen in der Entwicklung dieser Idee noch um einiges weiter und argumentieren für den Wert der »therapeutischen Justiz«. Gerichtsakteure sollten nicht nur für eine gute Kommunikation im Gerichtssaal sorgen, sondern sie sollten in ihrer Arbeit auch ein psychologisches Bewusstsein an den Tag legen und die Begegnung im Gerichtssaal dafür nutzen, psychologische Veränderungen bei Einzelpersonen zu unterstützen. Winick und Wexler halten fest, dass viele Gerichte heutzutage mit Problemen befasst sind, die sie vor zehn oder zwanzig Jahren nicht im gleichen Ausmaß betroffen haben - häusliche Gewalt, Kindesmisshandlung und Vernachlässigung, Geisteskrankheit und Substanzenmissbrauch. Die Gerichte mussten oder müssen sich erst - wie Winick und Wexler meinen - daran anpassen, dass sie Problemlöser und nicht nur juristische Schiedsrichter sind, wenn sie in ihrem Tun wirklich voll und ganz »erfolgreich« sein wollen.

Richter müssten, wie sie für kartellrechtliche Fälle über Ökonomie Bescheid wissen müssen, sich für andere Fälle mit psychologische Prinzipien vertraut machen. Winick und Wexler nehmen an, dass das Recht (die Rollen und das Verhalten der Rechtsanwender, Rechtsvorschriften und Verfahrensregeln) »therapeutische« Effekte haben kann. Gerichte sollten, wenn möglich, in einer Art und Weise reformiert werden, die die potentiell therapeutischen Effekte des Rechts maximieren, und zwar ohne die Rechte zu verletzen, die Angeklagten im Verfahren zukommen. So sollten Richter ihren Interaktionen mit Angeklagten mehr Aufmerksamkeit widmen und psychologische Prinzipien beachten, um eine Verhaltensänderung der Angeklagten zu fördern. Dazu gehört Wissen aus den Bereichen der Verhaltensund Motivationstheorie. Der entsprechende Nutzen von Gerichtsverfahren kann durch die Prozessplanung maximiert werden. Der Verfahrensablauf kann z.B. so eingerichtet werden, dass Lernprozesse für schwierige Täter gefördert werden, indem sie als letzte angehört werden, so dass sie die Möglichkeit haben, die kooperativeren Beschuldigten zu beobachten und von ihnen zu lernen (Winick and Wexler, 2003: 108).

\section{Restorative justice}

Verfahrensgerechtigkeit (procedural justice) und therapeutische Justiz sind zwei gute Leittheorien für gerichtliche Intervention. Sie sind bereits an Problemlösungs- oder spezialisierten Gerichten verankert wie auch in Programmen der wiederherstellenden Gerechtigkeit (restorative justice) verwirklicht oder in Justizprogrammen für Minderheiten (mit eigenständigen Rechtstraditionen, wie z.B. indigenen Urbevölkerungen). Derartige Programme in einer Vielzahl von Ländern streben danach, Rückfall zu vermindern, indem die Entscheidungsfindung bei Gericht direkt mit einem »Behandlungsprozess « verbunden wird, oder indem Dritte, wie die Familien der Täter und Opfer, in einen Prozess der Schadenswiedergutmachung eingebunden und die Anklagebehörden ermuntert werden, auf Strategien der lokalen Gemeinschaften zur Lösung der Kriminalitätsproblematik zurückzugreifen.

»Problemlösungsgerichte« bzw. spezialisierte Gerichte/Abteilungen befassen sich mit nur einem Typus von Fällen, z.B. Substanzmissbrauch, jugendlichen Tätern, häuslicher Gewalt. Vielleicht am besten bekannt sind die "Drogen-Gerichte . Das erste derartige Gericht wurde 1989 in Florida eingerichtet. Mit Stand September 2003 gab es in den Vereinigten Staaten 1.079 Drogengerichte, die in allen 50 Staaten, dem District of Columbia, Puerto Rico, Guam und zwei Bundesdistrikten arbeiten, wobei 419 weitere Drogengerichtsprogramme in Planung sind (OJP Drug Court Clearinghouse and Technical Assistance Project, 2003). In anderen Ländern wird mit ähnlichen Modellen experimentiert. $\mathrm{Zu}$ den wesentlichen Elementen eines Drogengerichts gehören: »die Einbindung von Drogentests in die Behandlung des Falles; die Herstellung einer nicht-gegnerschaftlichen Beziehung zwischen dem Angeklagten und dem Gericht; die Feststellung, welche Angeklagten Behandlung benötigen, sowie die nach der Verhaf- tung frühestmögliche Zuweisung zur Behandlung; Zugang zu einem Kontinuum von Behandlungsund Rehabilitiationsangeboten; die Überwachung der Abstinenz durch häufige, verpflichtende Drogentests; die Etablierung einer koordinierten Strategie für die Reaktionen der Drogengerichte auf die Einhaltung der Vorgaben durch die Programmteilnehmer; die Aufrechterhaltung der justizielle Interaktion mit jedem Drogengerichtsklienten; Monitoring und Evaluation der Programmziele und die Messung ihrer Effektivität; fortlaufende interdisziplinäre Ausbildung um effektive Planung, Implementierung und Tätigkeit der Drogengerichte zu gewährleisten und das Knüpfen von Partnerschaften zwischen Drogengerichten, öffentlichen Einrichtungen und Organisationen der örtlichen Gesellschaft, um lokale Unterstützung zu schaffen und die Effektivität der Drogengerichte zu verbessern «. (The National Association of Drug Court Professionals, 1997). Kürzlich durchgeführte Evaluationen (Gottfredson, Najaka and Kearley, 2003; Rempel et al., 2003) zeigen hinsichtlich der Reduktion von Rückfall vielversprechende Ergebnisse.

Wiederherstellende Gerechtigkeit (restorative justice) ist sowohl eine Theorie als auch eine Praxis, leidet aber am Fehlen einer gemeinsamen Definition. Sie hat sich aus den Justiztraditionen von Urbevölkerungen, wie denen von Australien oder Kanada, entwickelt ${ }^{4}$ und strebt nach einer Reintegration von Tätern und der Heilung von Opfern durch Gruppenprozesse. Diese Prozesse werden im allgemeinen von einem Moderator geleitet, finden in der Phase des Hauptverfahrens im Gericht oder auch außerhalb statt und streben nach Verständnis und Ausgleich. Wiederherstellende Gerechtigkeit baut weitgehend auf die Erfahrung von Scham, in einem Kontext von Versöhnung, wobei aber auch Anforderungen an die persönliche Verantwortung gestellt werden, die sich so sehr auf das Verhalten in der Zukunft wie auf das in der Vergangenheit richten. Wiederherstellende Gerechtigkeit ist auf der ganzen Welt en vogue und hat sogar in die Resolutionen der Vereinten Nationen zur Strafrechtsreform Eingang gefunden. Einen guten Überblick über Programme und Forschung findet man bei Hoyle und Young (2002).

\section{Conclusio: Die Rolle von Forschung und Evaluation im Court Management}

Abschließend lässt sich sagen, dass Court Management auf wachsendes Interesse vieler professioneller Rechtsanwender, Entwicklungsspezialisten und Forscher stößt. Dennoch liegt die Gerichtsforschung noch hinter anderen Bereichen der Strafjustizforschung zurück. Ein auffallendes Beispiel ist der Mangel an Forschung über die Anklage (National Research Council, 2001). Es ist ein Irrtum, dass wir erst eine Gerichtsreform bewerkstelligen oder eine neue Managementstruktur implementieren müssten, bevor man zur Evaluation übergehen kann. Aber selbst bei den Innovatio- 
nen im Bereich des Court Management verlangen die nationalen und internationalen Auftrag- und Geldgeber oft keine rigorosen Evaluationsdesigns. Deshalb ist es schwierig, Vergleiche über verschiedene Gerichtsbarkeiten und Staaten hinweg zu treffen und gut funktionierende Praktiken $\mathrm{zu}$ identifizieren. Die von Dakolias und Said (1999) vorgeschlagene Strategie von Pilotgerichten, in Verbindung mit einem von Anfang an rigorosen Evaluationsdesign, ist ein überzeugender Weg, Entwicklung und Evaluation im Rahmen von Aktionsforschung zu verbinden. Praktiker und Evaluatoren in einem Pilotgericht $\mathrm{zu}$ vereinen, ist eine überzeugende Methode, Veränderungen herbeizuführen und auch zu dokumentieren.

Dieser kurze Überblick hat gezeigt, dass an die Gerichte wachsende Erwartungen gestellt werden, beginnend bei ihrer Aufgabe als gute Rechtseinrichtungen, über ihre Rolle als gute Dienstleister, bis zu ihrer Aufgabe bei der Reduktion von Rückfälligkeit. Ohne gute Führung und gutes Management - in Verbindung mit passenden Ressourcen und guten Daten - wird es für Gerichte schwer sein, sich in Zukunft in diesem Ozean von Anforderungen zu »vermarkten«.

\section{Bibliography}

Bendala García, Rosa (2003) »Organización y Gestión de los Órganos Judiciales. « [Organization and Management of Courts ] Curso de Técnicas de Reforma Judicial [Course on Techniques of Judicial Reform]. Getafe, Madrid, Spain: Universidad Carlos III. June 11.

Comisión Andina de Juristas (2002) Reforma Judicial en la Región Andina: "¿Qué se ha hecho, dónde estamos, adónde vamos? « Lima, Perú: CAJ, Available at: http://www.cajpe.org.pe

Court Service (2003) Court Service 2002-2003 Annual Report and Accounts. London: Court Service. Available at: http://www.courtservice.gov. uk/about_us/our_performance/annual_report_02_03_contents.htm

Dakolias, María and Said, Javier (1999) »udicial Reform. A Process o Change Through Pilot Courts. «Washington, D.C.: World Bank. Available at: http://wwwwds.worldbank.org/servlet/WDS_IBank_Servlet?pcont=details\&eid=000094946_0004200546515

Derr, Kevin Lee (2001)»Evolution of Court Management «. In Dupont-Morales, M.A., Hooper, Michael K. and Schmidt, Judy H. (Eds.) »Handbook of Criminal Justice Administration.» New York: Marcel Dekker.

Gottfredson, Denise (2003) »Effectiveness of Drug Treatment Courts: Evidence from a Randomized Trial." Criminology and Public Policy. 2/2(March):171-196.

Hoyle, Carolyn and Young, Richard (2002) »Restorative Justice: Assessing the Prospects and Pitfalls«. Pp. 525-548 in McConville, Mike and Wilson, Geoffrey (Eds.) The Handbook of the Criminal Justice Process. Oxford: Oxford University Press.

Keilitz, Ingo (2000) »Standards and Measures of Court Performance. «In National Institute of Justice, Criminal Justice 2000. Washington, D.C.: Department of Justice, National Institute of Justice.

Mora Mora, Luis Paulino (2003) »La gestión judicial: Una evolución hacia la eficiencia y la inclusión« [Court management: Towards Efficiency and Inclusión«]. Presentation at the Third Regional Conference on Justice and Development in Latin America and the Caribeean. Inter-American Development Bank. Quito, Ecuador, 24- 26 July.
National Association for Court Management (2003) Core Competencies Curriculum Guidelines. Available at http://www.nacmnet.org/CCCG/cccg_CoreCompetencies.html.

National Research Council (2001) »What's Changing in Prosecution? Report of a Workshop «. Committee on Law and Justice, Philip Heymann and Carol Petrie, Editors. Division of Behavioral and Social Sciences and Education. Washington, D.C.: National Academy Press.

OJP Drug Court Clearinghouse and Technical Assistance Project (2003) Summary of Drug Court Activity by State and County. November 7. Washington, D.C.: American University, OJP Drug Court Clearinghouse and Technical Assistance Project. http://www.american.edu/academic.depts/spa/justice/publications/drgchart $2 k . p d f$

Ostrom, Brian J. and Hanson, Roger A. (1999) »Efficiency, Timeliness and Quality: A New Perspective from Nine State Criminal Trial Courts «. Williamsburg, VA: National Center for State Courts.

Pastor, Santos (1993) »jAh de la Justicia! Política judicial y Economía «. Madrid: Civitas.

Pastor, Santos (2003a) »Métodos y herramientas económicas para la reforma judicial." [Methods and Tools from Economics for Judicial Reform] Curso de Técnicas de Reforma Judicial [Course on Techniques of Judicial Reform]. Getafe, Madrid, Spain: Universidad Carlos III. June 10.

Pastor, Santos (2003b) »Los nuevos sistemas de organización y gestión de la justicia: ¿mito o realidad? « [»New organizational and management systems in the justice sector: Myth or Reality? «] Presentation at the Third Regional Conference on Justice and Development in Latin America and the Caribbean. InterAmerican Development Bank. Quito, Ecuador, 2426 July.

Plotnikoff, Joyce and Richard Woolfson (2002) "Judges Case Management Perspectives: The Views of Opinion Formers and Case Managers «. London: Lord Chancellor's Department Research Secretariat.

Michael Rempel, Dana Fox-Kralstein, Amanda Cissner, Robyn Cohen, Melissa Labriola, Donald Farole, Ann Bader and Michael Magnani (2003) »The New York State Adult Drug Court Evaluation: Policies, Participants and Impacts. « Report submitted to the New York State Unified Court System and the Bureau of Justice Assistance. Available at: http://courtinnovation.org/pdf/drug_court_eval.pdf

Tauri, Juan (1999) »Explaining Recent Innovations in New Zealand's Criminal Justice System: Empowering Maori or Biculturalising the State? "Australian and New Zealand Journal of Criminology «. 32/2:152-167.

Taxman, Faye and Ellis, Lori (1999) »Expediting Court Dispositions: Quick Results, Uncertain Outcomes." Journal of Research in Crime and Delinquency 36/1(February):30-55.

The National Association of Drug Court Professionals (1997) Defining Drug Courts:

The Key Components. Washington, D.C.: U.S. Department of Justice, Office of Justice Programs, Drug Courts Program Office. http://www. ncjrs.org/html/ bja/define/dfdpdf.pdf

Tobin, Robert W. (1997) An Overview of Court Administration in the United States.

Williamsburg, Virginia (USA): National Center for State Courts. Available at: http://www. ncsconline.org/ D_Intl/PDFs/TOBIN_Court_Admin-English.pdf

Tyler, Tom R. (2003) »Procedural Justice, Legitimacy and the Effective Rule of Law. «Pp. 283-357 in Tonry, Michael (Ed.) Crime and Justice: A Review of Research. Volume 30. Chicago: University of Chicago Press.

Weisheit, Ralph A., Falcone, David N. and Wells, L. Edward (1996) »Crime and Policing in Rural and Small-Town America «. Prospect Heights, Illinois, USA: Waveland Press.

Winick, Bruce J. and Wexler, David B. (Eds.) (2003) "Judging in a Therapeutic Key: Therapeutic Jurispruden- ce and the Courts «. Durham, North Carolina, USA: Carolina Academic Press.

Rosemary Barberet, Ph.D., und Ramón y Cajal, Research Fellow, arbeiten am Institut für Politikwissenschaft und Soziologie der Universidad Carlos III. in Madrid, Spanien

\section{Anmerkungen:}

1 Anm. der Übersetzerin: Der Kürze halber wurde im übrigen Text von einer durchgehende Nennung von weiblichen wie männlichen Formen abgesehen. Alle Personenbezeichnungen beziehen sich auf Frauen und Männer gleichermaßen.

2 Das Court Service ist eine ausführende Behörde des Department of Constitutional Affairs. 3 Bis dato behandelt der überwiegende Teil der Forschung und erhältlicher Information $\mathrm{zu}$ Court Management Gerichtshöfe in Städten. Viel weniger ist über Justizsysteme im ländlichen Raum bekannt. Ich bin mir wohl bewußt dass ländliche Gerichtswesen schwierig zu managen sein können, weil räumliche Entfernungen und kleine Zahlen von Fällen es schwierig machen, Dienste zu konzentrieren oder spezialisierte Dienstleistungen anzubieten. Die Bekanntheit der Gerichtsakteure miteinander und mit den Klienten des Gerichts stellt eine große Herausforderung an Fairness und Gerechtigkeit dar (Weisheit, Falcone, and Wells, 1996).

4 Tauri (1999) bietet einen guten kritischen Überblick über die Beziehung zwischen den Justizpraktiken der Urbevölkerung der Maori und den Programmen wiederherstellender Gerechtigkeit im Strafjustizsystem von Neuseeland.

\section{WEBLIOGRAPHY}

Die folgenden Websites sind nützliche Quellen für mehr Information über Court Management und Gerichtsreform.

Center for Court Innovation http://www.courtinnovation.org

Drug Court Clearinghouse and Technical Assistance Project http://www.american.edu/academic. depts/spa/justice/drugcourts.html

CEJA (Centro de Estudios de Justicia de las Américas) www.cejamericas.org

Inter-American Development Bank www.iadb.org

International Network on Therapeutic Jurisprudence http://www.therapeuticjurisprudence.org

National Association for Court Management http://www.nacmnet.org

National Center for State Courts http://www.ncsconline.org

NCJRS - U.S. clearinghouse for criminal justice research, section on the courts: http://virlib.ncjrs.org/Courts.asp

Restorative Justice online: http://www.restorativejustice.org

World Bank http://web.worldbank.org 